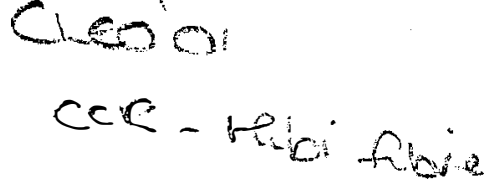

\title{
Single-polarization laser operation in polarization-maintaining single-polarization pumped erbium-doped fiber
}

\author{
J. K. Sahu, C. C. Renaud, J. Nilsson, W. A. Clarkson \\ Optoelectronics Research Centre, University ofSouthampton, Southampton S017 IBJ, UK \\ S. A. Alam, and A. B. Grudinin \\ SouthamptonPhotonics, Phi House, Enterprise Road, Chilworth SciencePark, Southampton S016 7NS. UK
}

We demonstrate a linearly polarized fiber laser in a polarization-maintaining erbium-doped fiber pumped by linearly polarized light. The laser output polarization followed the pump polarization when this was aligned on either of the fiber's principal axes.

\section{Summary}

We utilize polarization-dependent gain to generate single-polarization laser operation in a polarization-maintaining erbium-doped fiber laser (PM EDFL). The PM EDFL was pumped by linearly polarized light. The output polarization from the EDFL was co-linear with the pump when the pump was polarized along either of the principal axes of the PMEDFL.

Polarization-dependent gain (PDG) in erbium-doped fiber amplifiers (EDFAs) has been studied because of its importance in optical telecommunication [1-5]. It is typically quite small, e.g., $0.05-0.1 \mathrm{~dB}$. One source of PDG is the pump polarization: A linearly polarized pump may preferably amplify a parallel linear polarization. While EDFAs are typically pumped by linearly polarized diodes, the linear polarization is not maintained through the EDFA. Furthermore, a large magnetic dipole component of optical transitions in $\mathrm{Er}^{3+}$-ions makes them relatively insensitive to polarization. Still, a polarization-dependence of $20 \%$ of the cross-section for the ${ }^{4} I_{13 / 2} \rightarrow{ }^{4} I_{15 / 2}$ transition was determined in [2]. Such a large polarization-dependence is enough for single-polarization laser operation. With a polarization-maintaining fiber a linear pump polarization may remain throughout the fiber.

Nevertheless, as far as we know, no single-polarization EDFL of this kind has been reported in the literature.

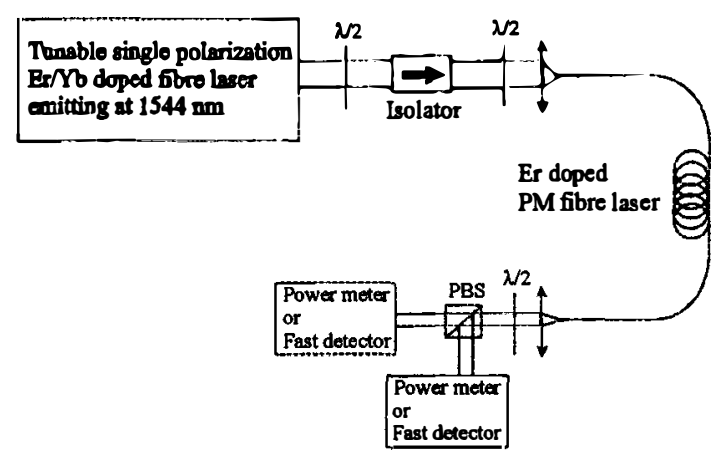

Fig. 1. Experimental set-up

Figure 1 depicts our experimental set-up. A wavelength-tunable linearly polarized cladding-pumped erbiumytterbium co-doped iber laser (EYDFL) pumped our PM EDFL. The EYDFL operated at $1544 \mathrm{~nm}$. Thus, the PM EDFL was pumped intra-band near its emission wavelength. An optical isolator separated the EYDFL from the PM EDFL. A $\lambda / 2$ waveplate adjusted the polarization-angle of the pump light launched into the PMEDFL. The perpendicularly cleaved ends of the PMEDFL generated $4 \%$ reflection feedback for the laser. We ensured that there was no feedback from other parts of the set-up. A polarizer at the far end of the PM EDFL analyzed its polarization characteristics. The PANDA-type PMEDFL was manufactured in-house. It was actually ytterbium-sensitized, but with intra-band pumping, the ytterbium does not take part in the transition processes. It had a small-signal absorption of $\sim 4 \mathrm{~dB} / \mathrm{m}$ at the pump wavelength. 


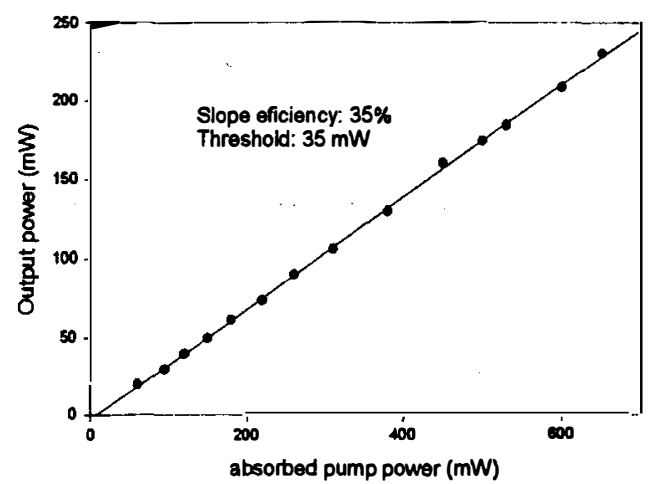

Fig. 2. Laser power characteristics. These did not change noticeably with pump polarization angle.

Figure 2 shows the double-ended laser characteristics of our PMEDFL. The emission wavelength was $1568 \mathrm{~nm}$. The slope efficiency of the PM EDFL was relatively poor, around $34 \%$ with respect to absorbed power. We believe the reason for this is the close proximity of pump and signal wavelengths, as well as a less-than-perfect gain medium. A double-clad non-PM fiber pulled from the same preform as the PM EDF had a slope efficiency of only $28 \%$, when cladding pumped at $915 \mathrm{~nm}$. We believe that the polarization-maintaining details of our EDFL did not further degrade its efficiency.

When the pump polarization was aligned with a principal axis of the PM EDFL, it would lase on the same polarization. When the pump polarization was rotated to the other principal axis, the PM EDFL output would follow. This was true for all pump powers we could achieve. It demonstrates that PDG induced by the pump polarization dominates over other sources of PDG, including polarization hole-buming. While single-polarization lasing leads to polarization hole-buming, this effect may be offset by the stronger PDG induced by a stronger pump, in which case single-polarization lasing can be maintained at power levels beyond those obtained here.

Single-polarization lasing was maintained over a range of pump polarization angles of $\sim \pm 5^{\circ}$. For larger pump polarization angles, the PMEDFL would operate on both polarizations. It would rapidly switch from one polarization to the other, as exemplified in Fig. 3. The power characteristics did not deviate from those illustrated in Fig. 2.

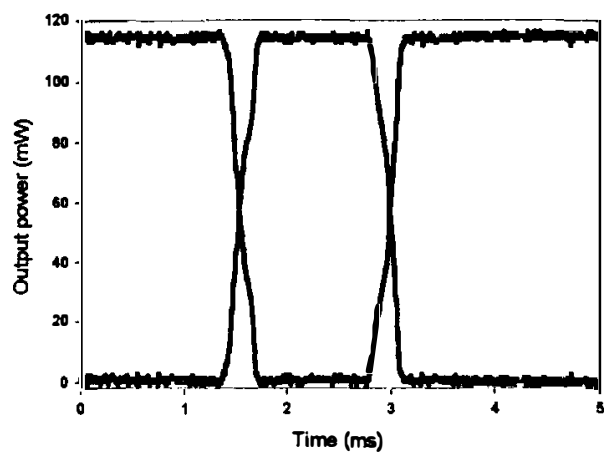

Fig. 3. Polarizetion switching of PM EDFL when pump polarization is not aligned on the fiber's principal axes. The two curves show the power in the main axis of the fibre as a fimction of time.

1. E. J. Greer, D. J. Lewis, and W. M. Macauley, "Polarisation dependent gain in erbium-doped fibre amplifiers", Electron. Lett. 30, 46-47 (1994)

2. A. J. Poustie, "Polarization cross saturation in an $\mathrm{E}^{3+}$-doped fiber ring laser", Opt. Lett. 20, 1868-1870 (1995)

3. P. F. Wysocli and V. Mazurczyk, "Polarization-dependent gain in erbium-doped fiber amplifiers: computer model and approximate formulas", J. Lightwave Technol. 14, 572-584 (1996)

4. K. Himeno, T. Sakai, S. Tomita, M. Sawada, A. Wada, and R. Yamauchi, "Polarization-maintaining erbium-doped optical fibers", Fujikura Technical Review (1997)

5. L. J. Wang, J. T. Lin, and P. Ye, "Analysis of polarization-dependent gain in fiber amplifiers", IEEE J. Quantum Electron. 34, 413-418 (1998) 